\title{
Relação da potência aeróbica máxima e da força muscular com a economia de corrida em atletas de endurance*
}

\author{
Luiz Guilherme Antonacci Guglielmo, Camila Coelho Greco e Benedito Sérgio Denadai
}

\section{RESUMO}

O objetivo deste estudo foi analisar a relação da potência aeróbica máxima e da força muscular (força isotônica máxima e força explosiva de salto vertical) com a economia de corrida (EC) em atletas de endurance. Vinte e seis corredores do sexo masculino $(27,9 \pm 6,4$ anos; $62,7 \pm 4,3 \mathrm{~kg} ; 168,6 \pm 6,1 \mathrm{~cm} ; 6,6 \pm 3,1 \%$ de gordura corporal) realizaram, em diferentes dias, as seguintes provas: a) teste incremental para a determinação do consumo máxi-

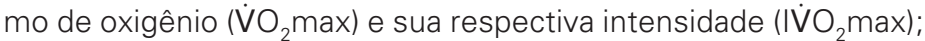
b) teste submáximo com velocidade constante para determinar a EC; c) teste de carga máxima no leg press; e d) altura máxima de salto com contramovimento (SV). O $\mathrm{VO}_{2} \max (63,8 \pm 8,3 \mathrm{ml} / \mathrm{kg} / \mathrm{min})$ foi significantemente correlacionado $(r=0,63$; $p<0,05)$ com a EC $(48,0 \pm 6,6 \mathrm{ml} / \mathrm{kg} / \mathrm{min})$. Por outro lado, a IVंO $\max (18,7 \pm 1,1 \mathrm{~km} / \mathrm{h})$, a força isotônica máxima $(230,3 \pm 41,2 \mathrm{~kg})$ e o SV $(30,8 \pm 3,8 \mathrm{~cm})$ não foram significantemente relacionados com a EC. Conclui-se que a potência aeróbica máxima explica em parte as variações interindividuais da EC em atletas de endurance. Entretanto, a força isotônica máxima e a força explosiva parecem não estar associadas com os valores de EC neste grupo de atletas.

\section{RESUMEN}

Relación de la potencia aeróbica máxima y de la fuerza muscular con la economía de le carrera en atletas de endurance

El objetivo de este estudio fué el de analizar la relación de la potencia aeróbica máxima y da la fuerza muscular (fuerza isotónica máxima y de la fuerza explosiva de salto vertical) con la economía de carrera (EC) en atletas de endurance. Veintiseis corredores de sexo masculino $(27,9 \pm 6,4$ años; $62,7 \pm 4,3 \mathrm{~kg} ; 168,6 \pm 6,1 \mathrm{~cm}$; $6,6 \pm 3,1 \%$ de grasa corporal) realizaron en diferentes días, los seguintes tests: a) test incremental para la determinación del consumo máximo de oxígeno $\left(\dot{V}_{2} \mathrm{max}_{2}\right.$ y su respectiva intensidad (IV $\mathrm{O}_{2}$ max); b) test submáximo con velocidad constante para determinar la $E C$; c) test de carga máxima como leg press y; d) altura máxima de salto con contramovimento (SV). El $\dot{V} O_{2} \max (63,8 \pm$ $8,3 \mathrm{ml} / \mathrm{kg} / \mathrm{min})$ fué significantemente correlacionado $(r=0,63 ; p<$ $0,05)$ con la $E C(48,0 \pm 6,6 \mathrm{~m} / \mathrm{kg} / \mathrm{min})$. Por otro lado, la $/ \dot{\mathrm{VO}}{ }_{2} \mathrm{max}$ $(18,7 \pm 1,1 \mathrm{~km} / \mathrm{h})$, la fuerza isotónica máxima $(230,3 \pm 41,2 \mathrm{~kg})$ y el SV $(30,8 \pm 3,8 \mathrm{~cm})$ no fueron significantemente relacionados con la EC. Se concluye que la potencia aeróbica máxima explica en parte las variaciones interindividuales de la EC en atletas de endurance. Entretanto, la fuerza isotónica máxima y la fuerza explosiva parecen no estar asociadas con los valores de EC en este grupo de atletas.

\footnotetext{
* Laboratório de Avaliação da Performance Humana - Unesp, Rio Claro. Apoio: Fapesp e CNPq.

Recebido em 8/12/04. 2a versão recebida em 19/1/05. Aceito em 23/1/05
}

Endereço para correspondência: B.S. Denadai, Laboratório de Avaliação da Performance Humana, IB, Unesp - Av. 24A, 1.515, Bela Vista - 13506900 - Rio Claro, SP, Brasil. E-mail: bdenadai@rc.unesp.br
Palavras-chave: Economia de corrida. Consumo máximo de oxigênio. Força mus-
cular.
Palabras-clave:
Economía de carrera. Consumo máximo de oxígeno. Fuerza mus-

\section{INTRODUÇÃO}

A economia de corrida (EC) pode ser definida como o custo de oxigênio $\left(\mathrm{V}_{2}\right)$ para uma dada velocidade submáxima de corrida(1). Alguns autores têm mostrado uma variabilidade interindividual bem elevada (> 15\%) na EC, mesmo entre indivíduos bem treinados e com valores similares de consumo máximo de oxigênio $\left(\dot{V}_{2} \max \right)^{(2)}$. Melhor EC (i.e., menor $\dot{V}_{2}$ para determinada velocidade de corrida) pode ser vantajosa, principalmente em provas de endurance, pois permitirá menor utilização fracional do $\dot{V}_{2}$ max, para qualquer intensidade submáxima de exercício. Em condições experimentais bem controladas, a EC apresenta boa reprodutibilidade, mostrando variação intra-individual de 1,5 a $5 \%{ }^{(3)}$.

Parte da variabilidade da EC tem sido associada a fatores antropométricos (distribuição da massa nos segmentos), fisiológicos (tipo de fibra muscular), biomecânicos e técnicos ${ }^{(4)}$. Interessantemente, alguns estudos realizados na corrida(5,6) e também no ciclismo ${ }^{(7)}$ têm verificado uma relação inversa do $\mathrm{V}_{2}$ max com a EC (corrida) e a eficiência (ciclismo). Esses resultados têm gerado uma série de debates ${ }^{(8,9)}$, indicando a necessidade de outros estudos que investiguem essa relação $\left(\mathrm{V}_{2}\right.$ max $x$ EC) em atletas com diferentes níveis de performance.

$\mathrm{O}$ treinamento intervalado de alta intensidade de característica aeróbica ( $5 x \sim 2,5 \mathrm{~min}$ a $100 \%$ do $\dot{\mathrm{V}} \mathrm{O}_{2}$ max), com uma ou duas sessões semanais, realizado durante quatro semanas, pode ser suficiente para a melhora da EC de corredores de fundo(10,11). Do mesmo modo, a adição do treinamento de força explosiva (treino pliométrico), durante nove semanas, melhorou a EC (8\%) e a performance $(3 \%)$ de corredores na distância de $5 \mathrm{~km}^{(12)}$. A adaptação neural (maior ativação neural das unidades motoras) e o aumento da capacidade de utilizar a energia elástica estocada no conjunto músculo-tendão têm sido apontados como os prováveis mecanismos que podem determinar a melhora da EC com o treino pliométrico ${ }^{(13)}$. Assim, poder-se-ia conjeturar que as características neuromusculares explicariam em parte a variabilidade interindividual da EC em corredores treinados. Entretanto, para o nosso conhecimento, nenhum estudo investigou a relação entre a força muscular e a EC em atletas. Com isso, o objetivo deste estudo foi analisar a relação da potência aeróbica máxima $\left(\dot{V}_{2} \mathrm{O}_{2} \mathrm{max}\right)$ e da força muscular (força isotônica máxima e força explosiva de salto vertical) com a EC em atletas de endurance.

\section{MATERIAL E MÉTODOS}

\section{Sujeitos}

Participaram deste estudo 26 corredores do sexo masculino $(27,9$ $\pm 6,4$ anos; $62,7 \pm 4,3 \mathrm{~kg} ; 168,6 \pm 6,1 \mathrm{~cm} ; 6,6 \pm 3,1 \%$ de gordura corporal) bem treinados em provas de meio-fundo e fundo do atletismo. Todos os corredores treinavam seis dias por semana, com um volume semanal que oscilava entre 70 a $90 \mathrm{~km}$. Todos os parti- 
cipantes conheceram os procedimentos do experimento e suas implicações (riscos e benefícios), através de um termo de consentimento escrito e explicado. O protocolo foi aprovado pelo Comitê de Ética em Pesquisa da instituição onde o experimento foi realizado (Protocolo 906).

\section{Delineamento experimental}

Todos os indivíduos estudados compareceram ao laboratório em quatro oportunidades diferentes, com um intervalo de cinco a sete dias entre o primeiro e o último comparecimento. Os indivíduos foram instruídos a não treinar exaustivamente no dia anterior à avaliação e compareceram alimentados e hidratados no dia do teste.

No primeiro comparecimento os indivíduos foram submetidos a um protocolo incremental na esteira rolante para a determinação do $\dot{\mathrm{V}} \mathrm{O}_{2}$ max e da intensidade correspondente ao $\dot{\mathrm{V}} \mathrm{O}_{2}$ max $\left(\mathrm{I} \dot{\mathrm{V}} \mathrm{O}_{2} \mathrm{max}\right)$, além de ter sido submetidos às mensurações antropométricas. No segundo dia, os atletas foram submetidos a um teste para a determinação da EC. No terceiro e quarto dias, realizaram, em ordem aleatória, um teste para avaliar a força explosiva de salto vertical (SV) e outro para a determinação de força isotônica máxima.

\section{Determinação do $\dot{V} \mathrm{O}_{2} \max$ e da $\mathrm{IV}_{\mathbf{V}} \max$}

$\mathrm{O} \dot{\mathrm{V}} \mathrm{O}_{2}$ max foi determinado utilizando-se um protocolo incremental em uma esteira rolante (/mbramed Millenuim Super ATL). A carga inicial foi de $12 \mathrm{~km} / \mathrm{h}$ ( $1 \%$ de inclinação), com incrementos de $1 \mathrm{~km} / \mathrm{h}$ a cada três minutos até à exaustão voluntária. Entre cada estágio houve um intervalo de 30 s para a coleta de sangue do lóbulo da orelha para a dosagem do lactato sanguíneo. $\mathrm{O} \mathrm{VO}_{2}$ foi mensurado respiração a respiração durante todo o protocolo a partir do gás expirado ( $\mathrm{K} 4 \mathrm{~b}^{2}$, Cosmed), sendo os dados reduzidos a médias de 15s. O $\dot{\mathrm{V}} \mathrm{O}_{2}$ max foi considerado como o maior valor obtido durante o teste nesses intervalos de 15s. Para considerarmos que durante o teste os indivíduos atingiram o $\dot{\mathrm{V}}_{2}$ max, foram adotados os critérios propostos por Taylor et al..(14) e Lacour et al.(15). A IV $\mathrm{V}_{2}$ max foi a menor velocidade em que o $\dot{\mathrm{V}} \mathrm{O}_{2}$ max foi alcançado e mantido por ao menos um minuto. Se o $\dot{V}_{2}$ max alcançado durante o estágio não fosse sustentado por um minuto, a velocidade do estágio anterior foi assumida como a IV $\mathrm{VO}_{2} \mathrm{max}^{(10)}$. O lactato sanguíneo foi determinado por um método eletroquímico (YSL 2300 STAT).

\section{Teste para determinar a economia de corrida (EC)}

Os corredores realizaram aquecimento por sete minutos a $12 \mathrm{~km} /$ h, seguido por um descanso de três minutos e, posteriormente, correram por mais oito minutos a $14 \mathrm{~km} / \mathrm{h}$. O $\mathrm{VO}_{2}$ foi medido entre o 6o e 7omin a $14 \mathrm{~km} / \mathrm{h}$, servindo como referência para a EC do atleta, que foi definida como a relação entre o $\mathrm{V}_{2}$ e a velocidade de corrida ${ }^{(1)}$.

\section{Determinação da força explosiva de salto vertical}

Os sujeitos foram submetidos a um teste de SV para a determinação da elevação do centro de gravidade em relação ao solo. A elevação do centro de gravidade do corpo foi determinada por meio do equipamento jump test (versão 1.1). A validade do equipamento já foi previamente determinada em comparação com uma plataforma de força ${ }^{(16)}$. Foi utilizada uma técnica de salto vertical com um movimento de preparação (contramovimento) em que é permitido ao executante realizar a fase excêntrica para a seguir executar a fase concêntrica do movimento. O indivíduo parte de uma posição em pé, com as mãos fixas na cintura e os pés paralelos e separados aproximadamente à largura dos ombros, e se movimenta para baixo flexionando as articulações do quadril, joelhos e tornozelos. A transição da primeira fase (descendente) para a fase seguinte (ascendente) acontece em um movimento contínuo, no qual as articulações são estendidas o mais rápido possível. Dessa forma, os mecanismos associados ao ciclo muscular estiramento-en- curtamento podem ser utilizados. Esse salto tem sua aplicação na determinação do nível de força explosiva dos membros inferiores (impulsão vertical).

Todos realizaram aquecimento com duração de 10 a 15min, o qual foi constituído por alongamentos e alguns saltos e, em seguida, os atletas realizaram cinco saltos no jump test com 30s de intervalo entre cada salto. A altura máxima foi considerada como a média aritmética dos três melhores saltos.

\section{Protocolo para a determinação da força isotônica máxima}

A força isotônica máxima foi determinada utilizando-se um protocolo de carga máxima (1RM) obtida durante a flexão e extensão dos joelhos por meio de um aparelho específico de musculação (leg-press $45^{\circ}$ ). Os atletas realizaram aquecimento antes do teste, constituído por alongamentos e três séries com 15 repetições (30\% da massa corporal) e com 60s de intervalo entre cada série. Após esse procedimento, a força isotônica máxima foi determinada como sendo a carga máxima na qual os atletas realizaram o movimento completo de flexão e extensão dos joelhos atingindo angulação de $90^{\circ}$ durante a fase excêntrica. Foram realizadas no máximo cinco tentativas no mesmo dia, com cinco minutos de intervalo entre cada repetição(17).

\section{Análise estatística}

Os dados estão expressos como média \pm desvio-padrão (DP). A relação da EC (variável dependente) com o $\dot{V} O_{2} \max$, a IV ${ }_{2} \max$, a força isotônica máxima e o SV (variáveis independentes), foi analisada pelo teste de correlação de Pearson. Em todos os testes foi adotado o nível de significância de $p \leq 0,05$.

\section{RESULTADOS}

A tabela 1 mostra os valores do $\dot{\mathrm{V}} \mathrm{O}_{2} \max _{\text {, da }} \mathrm{IV} \mathrm{V}_{2} \max _{\text {, da }} \mathrm{EC}$, da carga máxima e da altura máxima de salto.

TABELA 1

Valores médios \pm DP do consumo máximo de oxigênio $\left(\mathrm{V}_{2} \mathrm{max}_{\mathrm{m}}\right.$ e da sua respectiva intensidade (IV் $\mathrm{O}_{2} \mathrm{max}$ ), da economia de corrida (EC), da carga máxima (1 RM) e da altura máxima de salto (SV). N = 26

\begin{tabular}{|c|c|c|c|c|c|}
\hline & $\begin{array}{c}\dot{\mathrm{V}} \mathrm{O}_{2} \mathrm{max} \\
(\mathrm{ml} / \mathrm{kg} / \mathrm{min})\end{array}$ & $\begin{array}{c}\mathrm{IV}_{2} \max \\
(\mathrm{km} / \mathrm{h})\end{array}$ & $\begin{array}{c}\text { EC } \\
(\mathrm{ml} / \mathrm{kg} / \mathrm{min})\end{array}$ & $\begin{array}{c}1 \text { RM } \\
(\mathbf{k g})\end{array}$ & $\begin{array}{c}\text { SV } \\
(\mathrm{cm})\end{array}$ \\
\hline Média & 63,8 & 18,7 & 48,0 & 230,3 & 30,8 \\
\hline DP & 8,3 & 1,1 & 6,6 & 41,2 & 3,8 \\
\hline
\end{tabular}

$\mathrm{O} \dot{\mathrm{V}} \mathrm{O}_{2}$ max foi significantemente relacionado com a $\mathrm{EC}(\mathrm{r}=0,63$; $p<0,05)$ (figura 1). A IV $\mathrm{O}_{2} \max (r=-0,12 ; p>0,05)$, a altura máxima de salto $(r=0,13 ; p>0,05)$ e carga máxima expressa em valores absolutos $(r=-0,07 ; p>0,05)$ e relativos à massa corporal $(r=$ $0,04 ; p>0,05)$ não foram significantemente correlacionados com a EC.

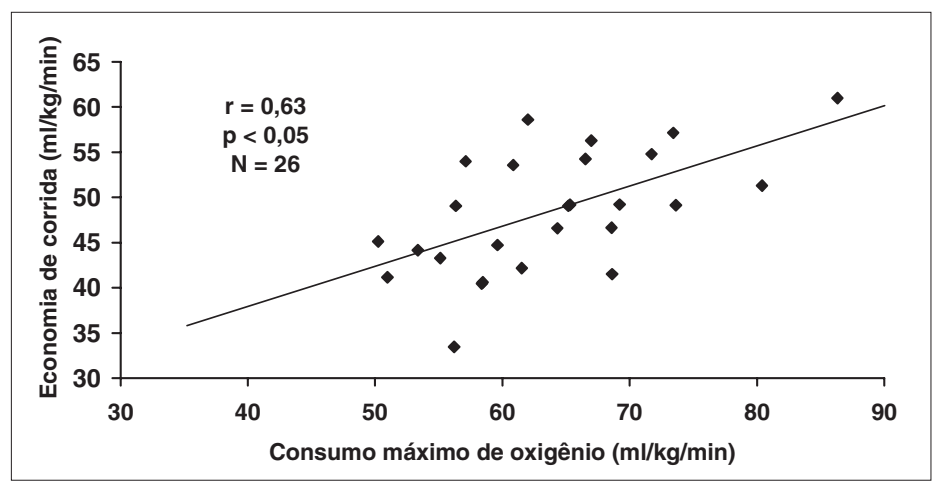

Fig. 1 - Relação entre o consumo máximo de oxigênio e a economia de corrida 


\section{DISCUSSÃO}

O principal o objetivo deste estudo foi analisar a relação entre a potência aeróbica máxima ( $\dot{V}_{2}$ max) e a força muscular (força isotônica máxima e força explosiva de salto vertical) com a EC em atletas de endurance. Concordando com estudos realizados anteriormente ${ }^{(5,6)}$, verificamos que a potência aeróbica máxima explica em parte as variações interindividuais da EC em atletas de endurance. Entretanto, a força isotônica máxima e a força explosiva parecem não estar associadas com os valores de EC nesse grupo de atletas.

A determinação da EC de atletas que participam de provas com predomínio aeróbico tem-se mostrado cada vez mais importante. Em atletas altamente treinados e com valores homogêneos de $\dot{\mathrm{V}}{ }_{2}$ max, a predição da performance aeróbica ${ }^{(13)}$ e o controle dos efeitos do treinamento aeróbico de alta intensidade ${ }^{(11)}$ ou com a adição do treino pliométrico(12), podem ser principalmente realizadas com a avaliação da EC. Assim, estudos que possam entender os fatores (fisiológicos, antropométricos e biomecânicos) que podem influenciar a EC são cada vez mais necessários.

Os valores de EC dos nossos sujeitos são bem similares aos encontrados em outros estudos que também analisaram atletas de meio-fundo e fundo, em velocidades similares às do presente estudo ${ }^{(10,18)}$. É importante destacar que, em condições experimentais bem controladas, a EC apresenta boa reprodutibilidade, mostrando variação intra-individual de 1,5 a 5\% (3). Além disso, a velocidade $(14 \mathrm{~km} / \mathrm{h})$ na qual o teste de EC foi realizado correspondeu em média a $75 \%$ VंO $\max _{2}$ afastando a possibilidade da existência do componente lento do $\dot{\mathrm{VO}}_{2}$, o qual poderia interferir nos valores de EC.

Pate et al.(6), analisando um grupo de corredores recreacionais ( $n=188)$, encontraram baixa correlação $(r=0,26)$, porém estatisticamente significante $(\mathrm{p}<0,001)$ entre o $\dot{\mathrm{VO}}_{2}$ max e o $\dot{\mathrm{VO}}_{2}$ submáximo durante a corrida com velocidade de $9,6 \mathrm{~km} / \mathrm{h}$. Com resultados bem semelhantes aos nossos, Morgan e Daniels ${ }^{(5)}$ verificaram em corredores bem treinados $\left(\mathrm{V}_{2} \mathrm{max}=75 \mathrm{ml} / \mathrm{kg} / \mathrm{min}\right)$ uma correlação moderada $(r=0,59 ; p<0,01)$ entre o $\dot{V}_{2}$ max e a EC. Pate et al.(6) propõem que parte da associação entre a EC e o $\dot{\mathrm{VO}}_{2}$ max pode ser explicada pela utilização dos diferentes substratos energéticos. Em seu estudo, os autores encontraram uma relação inversa $\left(r=-0,35 ; p=0,002\right.$ ) entre $\circ \dot{V}_{2}$ max e o $\mathrm{R}$ (quociente respiratório) obtido durante a corrida submáxima indicando que, nesta condição, os indivíduos com maior $\dot{V}_{2}$ max utilizaram maior percentual de gordura. Como o metabolismo da gordura requer uma quantidade maior de $\mathrm{O}_{2}$ por unidade de energia produzida, os autores propõem que os corredores com um $\mathrm{VO}_{2}$ max mais elevado necessitaram de um $\dot{V}_{2}$ submáximo maior durante a corrida. Devese salientar que, neste estudo, a intensidade da corrida $(9,6 \mathrm{~km} / \mathrm{h})$ correspondeu em média a $68 \%$ do $\dot{V}_{2}$ max, apresentando, porém, grande variação individual (46-91\% do $\dot{\mathrm{V}}_{2}$ max). Por outro lado, na investigação conduzida por Morgan e Daniels ${ }^{(5)}$, a variação da intensidade relativa da corrida foi bem menor (3-4\%), fazendo com que o $\mathrm{R}$ e, conseqüentemente, a utilização dos substratos, fosse responsável por uma pequena variabilidade da EC (<4\%), questionando a influência da participação desse fator sobre a EC em indivíduos treinados.

Em função disso, Morgan e Daniels ${ }^{(5)}$ propuseram que a relação entre o $\dot{V}_{2}$ max e a EC poderia ser explicada pelas diferenças nas distribuições da massa corporal nos segmentos, particularmente nos membros inferiores. Dentro dessa hipótese, os autores propõem que os sujeitos que apresentam percentual maior de sua massa corporal nos membros inferiores teriam $\dot{\mathrm{V}}_{2}$ max mais elevado, tendo em vista maior massa muscular ativa durante a corrida. Com isso, esses mesmos sujeitos podem apresentar $\dot{V}_{2}$ submáximo maior para acelerar seus membros inferiores, possuindo assim gasto energético mais elevado. É importante salientar que essa hipótese ainda não foi testada de forma apropriada.
No ciclismo, onde os efeitos da massa corporal sobre o gasto energético são menos importantes, principalmente quando a variação da composição corporal é pequena, Lucia et al.(7) também encontraram relação significante $(r=0,65 ; p<0,05)$ entre o gasto energético submáximo e o $\dot{\mathrm{VO}}_{2}$ max em ciclistas de elite. Lucia et al. ${ }^{(7)}$ propõem que o menor $\mathrm{V}_{2}$ max apresentado pelos seus ciclistas de alto rendimento é compensado pela maior eficiência, o que acabaria gerando valores de $\mathrm{IVO}_{2}$ max mais homogêneos. Ou seja, a seleção imposta pelo esporte de alto nível permitiria que ciclistas com valores relativamente baixos de $\mathrm{V}_{2}$ max alcançassem rendimento elevado, por possuírem maior eficiência. É importante lembrar que a $\mathrm{IVO}_{2}$ max é determinada principalmente pela interação do $\mathrm{V}_{2}$ max e da EC (corrida) ou eficiência (ciclismo), sendo melhor preditora da performance aeróbica, do que o $\dot{\mathrm{VO}}_{2}$ max ou a EC isoladamente ${ }^{(19)}$.

Nossos dados parecem confirmar, pelo menos em parte, a hipótese apontada por Lucia et al.(7). Primeiro, não foi verificada correlação significante entre a $I \dot{V} O_{2}$ max e a $E C(r=-0,12)$. Segundo, a variabilidade interindividual da $\mathrm{IVO}_{2} \max (6 \%)$ foi bem menor do que o $\dot{\mathrm{V}} \mathrm{O}_{2} \max (13 \%)$ e a $\mathrm{EC}(12,5 \%)$

A velocidade máxima de encurtamento do sarcômero (Vmax) da fibra do tipo II em humanos é 3-5 vezes maior do que aquela encontrada na fibra do tipo ${ }^{(20)}$. A eficiência muscular, definida como sendo a quantidade de trabalho realizado pela fibra muscular em relação ao seu gasto energético, é maior quando a velocidade de contração é de aproximadamente $1 / 3$ da Vmax para os dois tipos de fibra(20). Assim, quando as contrações são realizadas isometricamente ou em baixas velocidades, as fibras do tipo I são mais eficientes do que as do tipo II $^{(21)}$. A relação entre \% do tipo de fibra muscular e EC apresenta ainda informações contraditórias. Williams e Cavanagh(22) não encontraram correlação entre \% de fibra do tipo I e $\dot{\mathrm{V}} \mathrm{O}_{2}$ submáximo, em corredores com grande variabilidade na EC. Do mesmo modo, Kyrolainen et al. ${ }^{(18)}$ não encontraram correlação significante entre a distribuição percentual do diferentes tipos de fibras e a EC em um grupo de corredores de meiadistância com valores homogêneos de EC. Por outro lado, Bosco et al. ${ }^{(23)}$ encontraram correlação positiva $(r=0,60 ; p<0,01)$ entre \% fibra tipo II e $\dot{\mathrm{V}} \mathrm{O}_{2}$ durante a corrida submáxima. Os autores propõem que as fibras do tipo I podem reter mais energia elástica, reduzindo o consumo energético do sistema oxidativo.

Alguns estudos têm verificado que o treinamento de força explosiva (treino pliométrico) associado ao treinamento de característica aeróbica pode melhorar a EC e a performance de corredores nas distâncias de $3 \mathrm{~km}^{(24)}$ e $5 \mathrm{~km}^{(12)}$. Como os índices fisiológicos que são associados com o rendimento aeróbico $\left(\dot{\mathrm{V}}_{2}\right.$ max e reposta de lactato ao exercício) não foram modificados nesses estudos, os autores atribuíram a melhora do rendimento às modificações que o treino pliométrico determinou na EC. A adaptação neural (maior ativação neural das unidades motoras) e o aumento da capacidade de utilizar a energia elástica estocada no conjunto músculo-tendão têm sido apontados como os prováveis mecanismos que podem determinar melhora da EC com o treino pliométrico(13)

Em nosso estudo, entretanto, não foi encontrada correlação significante da força isotônica máxima e do SV com a EC. É importante notar que os mecanismos que são apontados como responsáveis pela melhora da EC com o treino pliométrico estão potencialmente presentes no tipo de salto (contramovimento) avaliado em nosso estudo. Com isso, é possível conjeturar que as variáveis analisadas no presente estudo (força isotônica máxima e SV) não reflitam de modo completo as modificações determinadas pelo treino de força explosiva que levam à melhora da EC. Essa hipótese é confirmada, pelo menos em parte, por dados recentemente obtidos por Turner et al.(25), que verificaram melhora da EC com o treino pliométrico, sem, entretanto, encontrar modificações significantes no SV ou nas variáveis que poderiam indicar melhora na capacidade de estocar e utilizar a energia elástica. Deve-se assumir, entretanto, a limitação do nosso delineamento experimental, que também 
é freqüentemente encontrada em outros estudos ${ }^{(2,5)}$, de ter avaliado a $\mathrm{EC}$ em apenas uma velocidade $(14 \mathrm{~km} / \mathrm{h})$. Alguns autores têm sugerido que o comportamento interindividual da EC pode depender da velocidade analisada, ou seja, atletas mais econômicos em velocidades moderadas (14-15km/h) não são necessariamente os mais econômicos em velocidades mais elevadas (> 19km/h)(26). Nessas velocidades mais intensas, porém, a duração do teste e/ ou o momento em que os valores de $\dot{\mathrm{V}}_{2}$ são analisados podem interferir no cálculo da EC, em função da existência do componente lento do $\mathrm{V}_{2}$. Nessas condições, ainda, não é desprezível a existência de uma contribuição anaeróbica, que normalmente é ignorada, ou o método utilizado na sua determinação pode apresentar problemas de validade. De qualquer modo, parecem ser necessários mais estudos que procurem identificar os mecanismos responsáveis pela melhora da EC após a adição do treino pliométrico ao treino de característica aeróbica.

\section{CONCLUSÃO}

Com base nos resultados deste estudo, podemos concluir que a potência aeróbica máxima explica em parte as variações interindividuais da EC em atletas de endurance. Entretanto, a força isotônica máxima e a força explosiva parecem não estar associadas com os valores de EC nesse grupo de atletas.

Todos os autores declararam não haver qualquer potencial conflito de interesses referente a este artigo.

\section{REFERÊNCIAS}

1. Daniels JA. A physiologist's view of running economy. Med Sci Sports Exerc $1985 ; 17: 332-8$.

2. Morgan DW, Martin PE, Krahenbuhl GS, Baldini FD. Variability in running economy and mechanics among trained male runners. Med Sci Sports Exerc 1991; 23:378-83

3. Saunders PU, Pyne DB, Telford RD, Hawley JA. Reliability and variability of running economy in elite distance runners. Med Sci Sports Exerc 2004;36:1972-6.

4. Denadai BS. Índices fisiológicos de avaliação aeróbia: conceitos e aplicações. Ribeirão Preto, SP: BSD, 1999.

5. Morgan DW, Daniels JT. Relationship between $\dot{V}_{2}$ max and the aerobic demand of running in elite distance runners. Int J Sports Med 1994;15:426-9.

6. Pate RR, Macera CA, Bailey SP, Bartoli WP, Powell KE. Physiological, anthropometric, and training correlates of running economy. Med Sci Sports Exerc 1992; 24:1128-33.
7. Lucia A, Hoyos J, Perez M, Santalla A, Chicharro JL. Inverse relationship between $\dot{\mathrm{V}}_{2}$ max and economy/efficiency in world-class cyclists. Med Sci Sports Exerc 2002;34:2079-84.

8. Atkinson G, Davison R, Passfield L, Nevill AM. Could the correlation between maximal oxygen uptake and "economy" be spurious? Med Sci Sports Exerc 2003;35:1242-3

9. Noakes TD, Tucker R. Inverse relationship between $\dot{V}_{2}$ max and economy in world-class cyclists. Med Sci Sports Exerc 2004;36:1083-4.

10. Billat VL, Flechet $B$, Petit $B$, Muriaux G, Koralsztein, JP. Interval training at $\dot{V}_{2}$ max: effects on aerobic performance and overtraining markers. Med Sci Sports Exerc 1999;31:156-63.

11. Ortiz MJ, Denadai BS, Stella S, Mello MT. Efeitos do treinamento aeróbio de alta intensidade sobre a economia de corrida em corredores de endurance. Rev Bras Cienc Mov 2003;11:51-4.

12. Paavolainen L, Hakkinen K, Hamalainen I, Nummela A, Rusko H. Explosivestrength training improves $5-\mathrm{km}$ running time by improving running economy and muscular power. J Appl Physiol 1999;86:1527-33.

13. Saunders PU, Pyne DB, Telford RD, Hawley JA. Factors affecting running economy in trained distance runners. Sports Med 2004;34:465-85. Review.

14. Taylor HL, Buskirk ER, Henschel A. Maximal oxygen intake as an objective measure of cardiorespiratory performance. J Appl Physiol 1955;8:73-80.

15. Lacour JR, Padilla-Magunacelaya S, Chatard JC, Arsac L, Barthelemy JC. Assessment of running velocity at maximal oxygen uptake. Eur J Appl Physiol 1991; 62:77-82.

16. Mil-Homens P. Relações entre a altura ideal de queda do ressalto e a impulsão vertical absoluta e relativa. Motricidade Humana 1987;3:45-65.

17. McCarthy JP, Agre JC, Graf BK, Pozniak MA, Vailas AC. Compatibility of adaptive responses with combining strength and endurance training. Med Sci Sports Exerc 1995;27:429-36

18. Kyrolainen H, Kivela R, Koskinen S, McBride J, Andersen JL, Takala T, et al. Interrelationships between muscle structure, muscle strength, and running economy. Med Sci Sports Exerc 2003;35:45-9.

19. Denadai BS, Ortiz MJ, Mello MT. Physiological indexes associated with aerobic performance in endurance runners: effects of race duration. Rev Bras Med Esporte 2004;10:405-7

20. Coyle EF. Integration of the physiological factors determining endurance performance ability. Exerc Sport Sci Rev 1995;23:25-63.

21. Wendt IR, Gibbs CL. Energy production of rat extensor digitorum longus muscle. Am J Physiol 1973;224:1081-86.

22. Williams KR, Cavanagh $P$. Relationship between distance running mechanics, running economy, and performance. J Appl Physiol 1987;63:1236-45.

23. Bosco C, Montanari G, Ribacchi R, Giovenali P, Latteri F, lachelli G, et al. Relationship between the efficiency of muscular work during jumping and the energetics of running. Eur J Appl Physiol 1987;56:138-43.

24. Spurrs RW, Murphy AJ, Watsford ML. The effect of plyometric training on distance running performance. Eur J Appl Physiol 2003;89:1-7.

25. Turner AM, Owings M, Schwane JA. Improvement in running economy after 6 weeks of plyometric training. J Strength Cond Res 2003;17:60-7.

26. Daniels J, Daniels N. Running economy of elite male and elite female runners Med Sci Sports Exerc 1992;24:483-9. 